\title{
Extended Jacobian Elliptic Function Expansion Method and Its Applications in Biology
}

\author{
Emad H. M. Zahran', Mostafa M. A. Khater ${ }^{2}$ \\ ${ }^{1}$ Department of Mathematical and Physical Engineering, College of Engineering Shubra, Benha University, \\ Benha, Egypt \\ ${ }^{2}$ Department of Mathematics, Faculty of Science, Mansoura University, Mansoura, Egypt \\ Email: mostafa.khater2024@yahoo.com
}

Received 26 January 2015; accepted 22 June 2015; published 25 June 2015

Copyright $@ 2015$ by authors and Scientific Research Publishing Inc.

This work is licensed under the Creative Commons Attribution International License (CC BY).

http://creativecommons.org/licenses/by/4.0/

(c) (i) Open Access

\begin{abstract}
In this work, an extended Jacobian elliptic function expansion method is proposed for constructing the exact solutions of nonlinear evolution equations. The validity and reliability of the method are tested by its applications to Dynamical system in a new Double-Chain Model of DNA and a diffusive predator-prey system which play an important role in biology.
\end{abstract}

\section{Keywords}

Extended Jacobian Elliptic Function Expansion Method, Dynamical System in a New Double-Chain Model of DNA, A Diffusive Predator-Prey System, Traveling Wave Solutions, Solitary Wave Solutions

\section{Introduction}

The nonlinear partial differential equations of mathematical physics are major subjects in physical science [1]. Exact solutions for these equations play an important role in many phenomena in physics such as fluid mechanics, hydrodynamics, Optics, Plasma physics and so on. Recently many new approaches for finding these solutions have been proposed, for example, tanh-sech method [2]-[4], extended tanh-method [5]-[7], $\exp (-\varphi(\xi))$ [8][11], homogeneous balance method [12], F-expansion method [13]-[15], exp-function method [16] [17], trigonometric function series method [18], $\left(\frac{\boldsymbol{G}^{\prime}}{\boldsymbol{G}}\right)$-expansion method [19]-[22], Jacobi elliptic function method [23]-[26] and so on. 
The objective of this article is to apply the extended Jacobian elliptic function expansion method for finding the exact traveling wave solution of Dynamical system in a new Double-Chain Model of DNA and a diffusive predator-prey system which play an important role in biology and mathematical physics.

The rest of this paper is organized as follows: In Section 2, we give the description of the extended Jacobi elliptic function expansion method In Section 3, we use this method to find the exact solutions of the nonlinear evolution equations pointed out above. In Section 4, conclusions are given.

\section{Description of Method}

Consider the following nonlinear evolution equation

$$
F\left(u, u_{t}, u_{x}, u_{t t}, u_{x x}, \cdots\right)=0,
$$

where $F$ is polynomial in $u(x, t)$ and its partial derivatives in which the highest order derivatives and nonlinear terms are involved. In the following, we give the main steps of this method [23]-[26]

Step 1. Using the transformation

$$
u=u(\xi), \xi=x-c t,
$$

where $k$ and $c$ are the wave number and wave speed, to reduce Equation (1) to the following ODE:

$$
P\left(u, u^{\prime}, u^{\prime \prime}, u^{\prime \prime \prime}, \cdots\right)=0 \text {, }
$$

where $P$ is a polynomial in $u(\xi)$ and its total derivatives, while ' $=\frac{\mathrm{d}}{\mathrm{d} \xi}$ '.

Step 2. Making good use of ten Jacobian elliptic functions, we assume that (3) has the solutions in these forms:

$$
u(\xi)=a_{0}+\sum_{j=1}^{N} f_{i}^{j-1}(\xi)\left[a_{j} f_{i}(\xi)+b_{j} g_{i}(\xi)\right], i=1,2,3, \cdots
$$

With

$$
\begin{array}{ll}
f_{1}(\xi)=s n \xi, & g_{1}(\xi)=c n \xi, \\
f_{2}(\xi)=s n \xi, & g_{2}(\xi)=d n \xi, \\
f_{3}(\xi)=n s \xi, & g_{3}(\xi)=c s \xi, \\
f_{4}(\xi)=n s \xi, & g_{4}(\xi)=d s \xi, \\
f_{5}(\xi)=s c \xi, & g_{5}(\xi)=n c \xi, \\
f_{6}(\xi)=s d \xi, & g_{6}(\xi)=n d \xi,
\end{array}
$$

where $s n \xi, c n \xi, d n \xi$, are the Jacobian elliptic sine function, The jacobian elliptic cosine function and the Jacobian elliptic function of the third kind and other Jacobian functions which is denoted by Glaisher's symbols and are generated by these three kinds of functions, namely

$$
\begin{aligned}
& n s \xi=\frac{1}{s n \xi}, n c \xi=\frac{1}{c n \xi}, n d \xi=\frac{1}{d n \xi}, s c \xi=\frac{c n \xi}{s n \xi}, \\
& c s \xi=\frac{s n \xi}{c n \xi}, d s \xi=\frac{d n \xi}{s n \xi}, s d \xi=\frac{s n \xi}{d n \xi},
\end{aligned}
$$

That have the relations

$$
\begin{aligned}
& s n^{2} \xi+c n^{2} \xi=1, d n^{2} \xi+m^{2} s n^{2} \xi=1, n s^{2} \xi=1+c s^{2} \xi, \\
& n s^{2} \xi=m^{2}+d s^{2} \xi, s c^{2} \xi+1=n c^{2} \xi, m^{2} s d^{2}+1=n d^{2} \xi,
\end{aligned}
$$

With the modulus $m(0<m<1)$. In addition we know that

$$
\frac{\mathrm{d}}{\mathrm{d} \xi} s n \xi=c n \xi d n \xi, \frac{\mathrm{d}}{\mathrm{d} \xi} c n \xi=-s n \xi d n \xi, \frac{\mathrm{d}}{\mathrm{d} \xi} d n \xi=-m^{2} s n \xi c n \xi .
$$


The derivatives of other Jacobian elliptic functions are obtained by using Equation (8). To balance the highest order linear term with nonlinear term we define the degree of $u$ as $D[u]=n$ which gives rise to the degrees of other expressions as

$$
D\left[\frac{\mathrm{d}^{q} u}{\mathrm{~d} \xi^{q}}\right]=n+q, \quad D\left[u^{p}\left(\frac{\mathrm{d}^{q} u}{\mathrm{~d} \xi^{q}}\right)^{s}\right]=n p+s(n+q) .
$$

According the rules, we can balance the highest order linear term and nonlinear term in Equation (3) so that $n$ in Equation (4) can be determined.

In addition we see that when $m \rightarrow 1, s n \xi, c n \xi$, and $d n \xi$ degenerate as $\tanh \xi$, sech $\xi$, sech $\xi$, respectively, while when therefore Equation (5) degenerate as the following forms

$$
\begin{gathered}
u(\xi)=a_{0}+\sum_{j=1}^{N} \tanh ^{j-1}(\xi)\left[a_{j} \tanh (\xi)+b_{j} \operatorname{sech}(\xi)\right], \\
u(\xi)=a_{0}+\sum_{j=1}^{N} \operatorname{coth}^{j-1}(\xi)\left[a_{j} \operatorname{coth}(\xi)+b_{j} \operatorname{coth}(\xi)\right], \\
u(\xi)=a_{0}+\sum_{j=1}^{N} \tan ^{j-1}(\xi)\left[a_{j} \tan (\xi)+b_{j} \sec (\xi)\right], \\
u(\xi)=a_{0}+\sum_{j=1}^{N} \cot ^{j-1}(\xi)\left[a_{j} \cot (\xi)+b_{j} \csc (\xi)\right] .
\end{gathered}
$$

Therefore the extended Jacobian elliptic function expansion method is more general than sine-cosine method, the tan-function method and Jacobian elliptic function expansion method.

\section{Application}

\subsection{Example 1: Dynamical System in a New Double-Chain Model of DNA}

An attractive nonlinear model for the nonlinear science in the deoxyribonucleic acid (DNA). The dynamics of DNA molecules is one of the most fascinating problems of modern biophysics because it is at the basis of life. The DNA structure has been studied during last decades. The investigation of DNA dynamics has successfully predicted the appearance of important nonlinear structures. It has been shown that the non linearity is responsible for forming localized waves. These localized waves are interesting because they have the capability to transport energy without dissipation [27]-[35]. In Ref. [34] [35], it is given that a new double-chain model of DNA consists of two long elastic homogeneous strands which represent two poly nucleotide chains of the DNA molecule, connected with each other by an elastic membrane representing the hydrogen bonds between the base pair of the two chains. Under some appropriate approximation, the new double-chain model of DNA can be described by the following two general nonlinear dynamical system:

$$
\begin{gathered}
u_{t t}-c_{1}^{2} u_{x x}=\lambda_{1} u+\gamma_{1} u v+\mu_{1} u^{3}+\beta_{1} u v^{2}, \\
v_{t t}-c_{2}^{2} v_{x x}=\lambda_{2} v+\gamma_{2} u^{2}+\mu_{2} u^{2} v+\beta_{2} v^{3}+c_{0},
\end{gathered}
$$

where

$$
\begin{gathered}
c_{1}= \pm \frac{Y}{\rho} ; c_{2}= \pm \frac{F}{\rho} ; \lambda_{1}=\frac{-2 \mu}{\rho \sigma h}\left(c-l_{0}\right) ; \\
\lambda_{2}=\frac{-2 \mu}{\rho \sigma} ; \gamma_{1}=2 \gamma_{2}=\frac{2 \sqrt{2} \mu l_{0}}{\rho \sigma h^{2}} ; \mu_{1}=\mu_{2}=\frac{-2 \mu l_{0}}{\rho \sigma h^{3}} ; \\
\beta_{1}=\beta_{2}=\frac{4 \mu l_{0}}{\rho \sigma h^{3}} ; c_{0}=\frac{\sqrt{2} \mu\left(h-l_{0}\right)}{\rho \sigma},
\end{gathered}
$$

where $\rho, \sigma, Y$ and $F$ denote respectively the mass density, the area of transverse cross-section, the Young's 
modulus and tension density of each strand; $\mu$ is the rigidity of the elastic membrane; $h$ is the distance between the two strands, and $l_{0}$ is the height of the membrane in the equilibrium positive. In Equations (14) and (15), $u$ is the difference of the longitudinal displacements of the bottom and top strands, while $v$ is the difference of the transverse displacements of the bottom and top strands.

we first introduce the transformation

$$
v=a u+b,
$$

where $a$ and $b$ are constants, to reduce Equations (14) and (15) to the following system of equations:

$$
u_{t t}-c_{1}^{2} u_{x x}=u^{3}\left(\mu_{1}+\beta_{1} a^{2}\right)+u^{2}\left(2 \beta_{1} a b+a \gamma_{1}\right)+u\left(\lambda_{1}+b \gamma_{1}+\beta_{1} b^{2}\right),
$$

and

$$
u_{t t}-c_{2}^{2} u_{x x}=u^{3}\left(\mu_{2}+\beta_{2} a^{2}\right)+u^{2}\left(\frac{\gamma_{2}}{a}+\frac{\mu_{2} b}{a}+3 \beta_{2} a b\right)+u\left(\lambda_{2}+3 \beta_{2} b^{2}\right)+\frac{\lambda_{2} b}{a}+\frac{\beta_{2} b^{3}}{a}+\frac{c_{0}}{a} .
$$

Comparing Equations (18) and (19) and using (17) we deduce that $b=\frac{h}{\sqrt{2}}$ and $F=Y$. Now Equations (18) and (19) can be written as

$$
u_{t t}-c_{1}^{2} u_{x x}-A u^{3}-B u^{2}-C u=0
$$

where

$$
A=\frac{\alpha}{h^{3}}\left(-2+4 a^{2}\right) ; B=\frac{6 \sqrt{2} a \alpha}{h^{2}} ; C=\left(\frac{-2 \alpha}{l_{0}}+\frac{6 \alpha}{h}\right) ; \alpha=\frac{\mu l_{0}}{\rho \sigma} ; c_{1}^{2}=\frac{Y}{\rho} .
$$

The wave transformation $u(x, t)=u(\xi), \xi=k x+\omega t$, reduce Equation (20) to the following ODE:

$$
\left(\omega^{2}-k^{2} c_{1}^{2}\right) u^{\prime \prime}-A u^{3}-B u^{2}-C u=0,
$$

where $\omega^{2}-k^{2} c_{1}^{2} \neq 0$. Balancing $u^{\prime \prime}$ and $u^{3}$ yields, $N+2=3 N \rightarrow N=1$. Consequently, we have the formal solution:

$$
u=a_{0}+a_{1} s n+b_{1} c n,
$$

where $a_{0}, a_{1}$ and $b_{1}$ are constant such that $a_{1} \neq 0$ or $b_{1} \neq 0$. From (23), it is easy to see that

$$
\begin{gathered}
u^{\prime}=a_{1} c n d n-b_{1} s n d n, \\
u^{\prime \prime}=-m^{2} s n a_{1}+2 a_{1} s n^{3} m^{2}+2 m^{2} s n^{2} c n b_{1}-a_{1} s n-b_{1} c n .
\end{gathered}
$$

Substituting Equations (23) and (25) into Equation (22) and equating all coefficients of $s n^{3}, s n^{2} c n, s n^{2}$, sncn, $s n, c n, s n^{0}$ to zero, we obtain

$$
\begin{gathered}
2\left(\omega^{2}-k^{2} c_{1}^{2}\right) m^{2} a_{1}-A\left(-3 a_{1} b_{1}^{2}+a_{1}^{3}\right)=0, \\
2\left(\omega^{2}-k^{2} c_{1}^{2}\right) m^{2} b_{1}-A\left(3 a_{1}^{2} b_{1}-b_{1}^{3}\right)=0, \\
-A\left(-3 a_{0} b_{1}^{2}+3 a_{0} a_{1}^{2}\right)-B\left(a_{1}^{2}-b_{1}^{2}\right)=0, \\
-6 A a_{0} a_{1} b_{1}-2 B a_{1} b_{1}=0, \\
\left(\omega^{2}-k^{2} c_{1}^{2}\right)\left(-a_{1}-m^{2} a_{1}\right)-A\left(3 a_{0}^{2} a_{1}+3 a_{1} b_{1}^{2}\right)-2 B a_{0} a_{1}-C a_{1}=0, \\
-\left(\omega^{2}-k^{2} c_{1}^{2}\right) b_{1}-A\left(3 a_{0}^{2} b_{1}-b_{1}^{3}\right)-2 B a_{0} b_{1}-C b_{1}=0, \\
-A\left(a_{0}^{3}+3 a_{0} b_{1}^{2}\right)-B\left(a_{0}^{2}+b_{1}^{2}\right)-C a_{0}=0 .
\end{gathered}
$$

Solving the above system with the aid of Maple or Mathematica, we have the following solitary wave solution: 


\section{Case 1.}

$$
A=\frac{2 m^{2}\left(-\omega^{2}+k^{2} c_{1}^{2}\right)}{b_{1}^{2}}, B=0, C=-\omega^{2}+k^{2} c_{1}^{2}-2 m^{2} \omega^{2}+2 m^{2} k^{2} c_{1}^{2}, a_{0}=0, a_{1}=0, b_{1}=b_{1},
$$

Case 2.

$$
\begin{aligned}
& A=\frac{2 m^{2}\left(-\omega^{2}+k^{2} c_{1}^{2}\right)}{b_{1}^{2}}, B= \pm \sqrt{\frac{-\left(1+2 m^{2}\right)}{2}} \frac{-6 m\left(-\omega^{2}+k^{2} c_{1}^{2}\right)}{b_{1}}, \\
& C=-2\left(1+2 m^{2}\right)\left(-\omega^{2}+k^{2} c_{1}^{2}\right), a_{0}= \pm \sqrt{\frac{-\left(1+2 m^{2}\right)}{2}} \frac{b_{1}}{m}, a_{1}=0, b_{1}=b_{1},
\end{aligned}
$$

Case 3.

$$
\begin{aligned}
& A=\frac{-2 m^{2}\left(-\omega^{2}+k^{2} c_{1}^{2}\right)}{a_{1}^{2}}, B= \pm \sqrt{\frac{\left(1+m^{2}\right)}{2}} \frac{6 m\left(-\omega^{2}+k^{2} c_{1}^{2}\right)}{a_{1}}, \\
& C=-2\left(1+m^{2}\right)\left(-\omega^{2}+k^{2} c_{1}^{2}\right), a_{0}= \pm \sqrt{\frac{\left(1+m^{2}\right)}{2}} \frac{a_{1}}{m}, a_{1}=a_{1}, b_{1}=0,
\end{aligned}
$$

Sothat solution of Equation (22) has the form

\section{Case 1.}

$$
u(\xi)= \pm \sqrt{\frac{2 m^{2}\left(-\omega^{2}+k^{2} c_{1}^{2}\right)}{A}} c n .
$$

Case 2.

$$
u(\xi)= \pm \sqrt{\frac{-\left(1+2 m^{2}\right)}{2}} \frac{b_{1}}{m} \pm \sqrt{\frac{2 m^{2}\left(-\omega^{2}+k^{2} c_{1}^{2}\right)}{A}} c n
$$

Case 3.

$$
u(\xi)= \pm \sqrt{\frac{\left(1+m^{2}\right)}{2}} \frac{a_{1}}{m} \pm \sqrt{\frac{-2 m^{2}\left(-\omega^{2}+k^{2} c_{1}^{2}\right)}{A}} s n .
$$

\subsection{Example 2. A Diffusive Predator-Prey System}

Consider a system of two coupled nonlinear partial differential equations describing the spatio-temporal dynamics of a predator-prey system [36],

$$
\begin{aligned}
& u_{t}=u_{x x}-\beta u+(1+\beta) u^{2}-u^{3}-u v, \\
& v_{t}=v_{x x}+\kappa u v-m v-\delta v^{3} .
\end{aligned}
$$

where $\kappa, \delta, m$ and $\beta$ are positive parameters. The solutions of predator-prey system have been studied in various aspects [36]-[38]. The dynamics of the diffusive predator-prey system have assumed the following relations between the parameters, namely $m=\beta$ and $\kappa+\frac{1}{\sqrt{\delta}}=\beta+1$. Under there assumptions, Equation (39) can be rewritten in the form:

$$
\begin{aligned}
& u_{t}=u_{x x}-\beta u+\left(\kappa+\frac{1}{\sqrt{\delta}}\right) u^{2}-u^{3}-u v, \\
& v_{t}=v_{x x}+\kappa u v-\beta v-\delta v^{3} .
\end{aligned}
$$

We use the wave transformation $u(x, t)=u(\xi), \xi=x-c t$ to reduce Equation (40) to the following non- 
linear system of ordinary differential equations:

$$
\left\{\begin{array}{l}
u^{\prime \prime}+c u^{\prime}-\beta u+\left(\kappa+\frac{1}{\sqrt{\delta}}\right) u^{2}-u^{3}-u v=0, \\
v^{\prime \prime}+c v^{\prime}+\kappa u v-\beta v-\delta v^{3}=0,
\end{array}\right.
$$

where $c$ is a nonzero constant.

In order to solve Equation (41), let us consider the following transformation

$$
v=\frac{1}{\sqrt{\delta}} u
$$

Substituting the transformation (42) into Equation (41), we get

$$
u^{\prime \prime}+c u^{\prime}-\beta u+\kappa u^{2}-u^{3}=0 .
$$

Balancing $u^{\prime \prime}$ with $u^{3}$ in Equation (43) yields, $N+2=3 N \Rightarrow N=1$. Consequently, we get the same formal solution (23). Substituting (23)-(25) into (43), setting the coefficients of $\left(s n^{3}, s n^{2} c n, s n^{2}, s n c n, c n\right.$, $\left.s n, s n^{0}\right)$ to zero, we obtain the following under determined system of algebraic equations for $\left(a_{0}, a_{1}, b_{1}\right)$.

$$
\begin{gathered}
2 a_{1} m^{2}-a_{1}^{3}+3 a_{1} b_{1}^{2}=0, \\
2 m^{2} b_{1}-3 a_{1}^{2} b_{1}+b_{1}^{3}=0, \\
\kappa\left(a_{1}^{2}-b_{1}^{2}\right)-3 a_{0} a_{1}^{2}+3 a_{0} b_{1}^{2}=0, \\
2 \kappa a_{1} b_{1}-6 a_{0} a_{1} b_{1}=0, \\
-a_{1} m^{2}-a_{1}-\beta a_{1}+2 \kappa a_{0} a_{1}-3 a_{0}^{2} a_{1}-3 a_{1} b_{1}^{2}=0, \\
-b_{1}-\beta b_{1}+2 k a_{0} b_{1}-3 a_{0}^{2} b_{1}-b_{1}^{3}=0, \\
-\beta a_{0}+\kappa\left(a_{0}^{2}+b_{1}^{2}\right)-a_{0}^{3}-3 a_{0} b_{1}^{2}=0,
\end{gathered}
$$

solving Equations (44)-(50) using the maple or mathematica program to get solitary wave solution of equations we get

$$
m= \pm \sqrt{\frac{\beta}{2}-1}, \kappa= \pm 3 \sqrt{\frac{\beta}{2}}, a_{0}=\sqrt{\frac{\beta}{2}}, a_{1}= \pm \sqrt{\beta-2}, \omega=\omega, b_{1}=0
$$

So we get

$$
\begin{gathered}
u(\xi)=a_{0}+a_{1} s n \\
u(\xi)= \pm \sqrt{m^{2}+1} \pm \sqrt{2} m s n,
\end{gathered}
$$

when $m=1$ hyperbolic solution

$$
u(\xi)= \pm \sqrt{2}(1+\tanh (\xi))
$$

\section{Conclusion}

We establish exact solutions for the dynamics of DNA molecules which is one of the most fascinating problems of modern biophysics because it is at the basis of life. The DNA structure has been studied during last decades. The investigation of DNA dynamics has successfully predicted the appearance of important nonlinear structures and a system of two coupled nonlinear partial differential equations describing the spatio-temporal dynamics of a predator-prey system where the prey per capita growth rate is subject to the All effect. The extended Jacobian elliptic function expansion method has been successfully used to find the exact traveling wave solutions of some nonlinear evolution equations. As an application, the traveling wave solutions for Dynamical system in a new Double-Chain Model of DNA and a diffusive predator-prey system, which have been constructed using the 
extended Jacobian elliptic function expansion method. Let us compare between our results obtained in the present article with the well-known results obtained by other authors using different methods as follows: Our results of the system of shallow water wave equations and a diffusive predator-prey system, are new and different from those obtained in [34]-[38]. It can be concluded that this method is reliable and proposes a variety of exact solutions NPDEs. The performance of this method is effective and can be applied to many other nonlinear evolution equations.

\section{References}

[1] Ablowitz, M.J. and Segur, H. (1981) Solitions and Inverse Scattering Transform. SIAM, Philadelphia. http://dx.doi.org/10.1137/1.9781611970883

[2] Malfliet, W. (1992) Solitary Wave Solutions of Nonlinear Wave Equation. American Journal of Physics, 60, 650-654. http://dx.doi.org/10.1119/1.17120

[3] Malfliet, W. and Hereman, W. (1996) The tanh Method: Exact Solutions of Nonlinear Evolution and Wave Equations. Physica Scripta, 54, 563-568. http://dx.doi.org/10.1088/0031-8949/54/6/003

[4] Wazwaz, A.M. (2004) The tanh Method for Travelling Wave Solutions of Nonlinear Equations. Applied Mathematics and Computation, 154, 713-723. http://dx.doi.org/10.1016/S0096-3003(03)00745-8

[5] EL-Wakil, S.A. and Abdou, M.A. (2007) New Exact Travelling Wave Solutions Using Modified Extended tanh-Function Method. Chaos Solitons Fractals, 31, 840-852. http://dx.doi.org/10.1016/j.chaos.2005.10.032

[6] Fan, E. (2000) Extended tanh-Function Method and Its Applications to Nonlinear Equations. Physics Letters A, 277, 212-218. http://dx.doi.org/10.1016/S0375-9601(00)00725-8

[7] Wazwaz, A.M. (2007) The Extended tanh Method for Abundant Solitary Wave Solutions of Nonlinear Wave Equations. Applied Mathematics and Computation, 187, 1131-1142. http://dx.doi.org/10.1016/j.amc.2006.09.013

[8] Abdelrahman, M.A.E., Zahran, E.H.M. and Khater, M.M.A. (2014) Exact Traveling Wave Solutions for Power Law and Kerr Law Non Linearity Using the $\exp (-\varphi(\xi))$-Expansion Method. GJSFR, 14-F, Version 1.0.

[9] Abdelrahman, M.A.E. and Khater, M.M.A. (2015) The $\exp (-\varphi(\xi))$-Expansion Method and Its Application for Solving Nonlinear Evolution Equations. International Journal of Science and Research (IJSR), 4, 2143-2146.

[10] Abdelrahman, M.A.E., Zahran, E.H.M. and Khater, M.M.A. (2015) The $\exp (-\varphi(\xi))$-Expansion Method and Its Application for Solving Nonlinear Evolution Equations. International Journal of Modern Nonlinear Theory and Application, 4, 37-47. http://dx.doi.org/10.4236/ijmnta.2015.41004

[11] Abdelrahman, M.A.E. and Khater, M.M.A. (2015) Exact Traveling Wave Solutions for Fitzhugh-Nagumo (FN) Equation and Modified Liouville Equation. International Journal of Computer Applications, 113, 1-7.

[12] Wang, M.L. (1996) Exact Solutions for a Compound KdV-Burgers Equation. Physics Letters A, 213, 279-287. http://dx.doi.org/10.1016/0375-9601(96)00103-X

[13] Abdou, M.A. (2007) The Extended F-Expansion Method and Its Application for a Class of Nonlinear Evolution Equations. Chaos, Solitons \& Fractals, 31, 95-104. http://dx.doi.org/10.1016/j.chaos.2005.09.030

[14] Ren, Y.J. and Zhang, H.Q. (2006) A Generalized F-Expansion Method to Find Abundant Families of Jacobi Elliptic Function Solutions of the $(2+1)$-Dimensional Nizhnik-Novikov-Veselov Equation. Chaos, Solitons \& Fractals, 27, 959-979. http://dx.doi.org/10.1016/j.chaos.2005.04.063

[15] Zhang, J.L., Wang, M.L., Wang, Y.M. and Fang, Z.D. (2006) The Improved F-Expansion Method and Its Applications. Physics Letters A, 350, 103-109. http://dx.doi.org/10.1016/j.physleta.2005.10.099

[16] He, J.H. and Wu, X.H. (2006) Exp-Function Method for Nonlinear Wave Equations. Chaos, Solitons \& Fractals, 30, 700-708. http://dx.doi.org/10.1016/j.chaos.2006.03.020

[17] Aminikhad, H., Moosaei, H. and Hajipour, M. (2009) Exact Solutions for Nonlinear Partial Differential Equations via Exp-Function Method. Numerical Methods for Partial Differential Equations, 26, 1427-1433.

[18] Zhang, Z.Y. (2008) New Exact Traveling Wave Solutions for the Nonlinear Klein-Gordon Equation. Turkish Journal of Physics, 32, 235-240.

[19] Wang, M.L., Zhang, J.L. and Li, X.Z. (2008) The $\left(\frac{G^{\prime}}{G}\right)$-Expansion Method and Travelling Wave Solutions of Nonlinear Evolutions Equations in Mathematical Physics. Physics Letters A, 372, 417-423. http://dx.doi.org/10.1016/j.physleta.2007.07.051 
[20] Zhang, S., Tong, J.L. and Wang, W. (2008) A Generalized $\left(\frac{G^{\prime}}{G}\right)$-Expansion Method for the mKdv Equation with Variable Coefficients. Physics Letters A, 372, 2254-2257. http://dx.doi.org/10.1016/j.physleta.2007.11.026

[21] Aslan, I. and Turgut, Ö. (2009) Analytic Study on Two Nonlinear Evolution Equations by Using the $\left(\frac{G^{\prime}}{G}\right)$-Expansion Method. Applied Mathematics and Computation, 209, 425-429. http://dx.doi.org/10.1016/j.amc.2008.12.064

[22] Zahran, E.H.M. and Khater, M.M.A. (2014) Exact Solutions to Some Nonlinear Evolution Equations by Using $\left(\frac{G^{\prime}}{G}\right)$ Expansion Method. Jökull Journal, 64, 226-238.

[23] Dai, C.Q. and Zhang, J.F. (2006) Jacobian Elliptic Function Method for Nonlinear Differential Difference Equations. Chaos, Solitons \& Fractals, 27, 1042-1049. http://dx.doi.org/10.1016/j.chaos.2005.04.071

[24] Fan, E. and Zhang, J. (2002) Applications of the Jacobi Elliptic Function Method to Special-Type Nonlinear Equations. Physics Letters A, 305, 383-392. http://dx.doi.org/10.1016/S0375-9601(02)01516-5

[25] Liu, S., Fu, Z., Liu, S. and Zhao, Q. (2001) Jacobi Elliptic Function Expansion Method and Periodic Wave Solutions of Nonlinear Wave Equations. Physics Letters A, 289, 69-74. http://dx.doi.org/10.1016/S0375-9601(01)00580-1

[26] Zahran, E.H.M. and Khater, M.M.A. (2014) Exact Traveling Wave Solutions for the System of Shallow Water Wave Equations and Modified Liouville Equation Using Extended Jacobian Elliptic Function Expansion Method. American Journal of Computational Mathematics, 4, 455-463. http://dx.doi.org/10.4236/ajcm.2014.45038

[27] Aguero, M., Najera, M. and Carrillo, M. (2008) Non Classic Solitonic Structures in DNA's Vibrational Dynamics. International Journal of Modern Physics B, 22, 2571-2582. http://dx.doi.org/10.1142/S021797920803968X

[28] Gaeta, G. (1999) Results and Limitations of the Soliton Theory of DNA Transcription. Journal of Biological Physics, 24, 81-96. http://dx.doi.org/10.1023/A:1005158503806

[29] Gaeta, G., Reiss, C., Peyrard, M. and Dauxois, T. (1994) Simple Models of Nonlinear DNA Dynamics. La Rivista Del Nuovo Cimento, 17, 1-48. http://dx.doi.org/10.1007/BF02724511

[30] Yakushevich, L.V. (1987) Nonlinear Physics of DNA. Studio Biophysica, 121, 201.

[31] Yakushevich, L.V. (1989) Nonlinear DNA Dynamics: A New Model. Physics Letters A, 136, 413-417. http://dx.doi.org/10.1016/0375-9601(89)90425-8

[32] Yakushevich, L.V. (1998) Nonlinear Physics of DNA. Wiley and Sons, England.

[33] Peyrard, M. and Bishop, A. (1989) Statistical Mechanics of a Nonlinear Model of DNA Denaturation. Physical Review Letters, 62, 2755-2758. http://dx.doi.org/10.1103/PhysRevLett.62.2755

[34] Kong, D.X., Lou, S.Y. and Zeng, J. (2001) Nonlinear Dynamics in a New Double Chain-Model of DNA. Communications in Theoretical Physics, 36, 737-742. http://dx.doi.org/10.1088/0253-6102/36/6/737

[35] Alka, W., Goyal, A. and Kumar, C.N. (2011) Nonlinear Dynamics of DNA-Riccati Generalized Solitary Wave Solutions. Physics Letters A, 375, 480-483. http://dx.doi.org/10.1016/j.physleta.2010.11.017

[36] Petrovskii, S.V., Malchow, H. and Li, B.L. (2005) An Exact Solution of a Diffusive Predator-Prey System. Proceedings of the Royal Society A, 461, 1029-1053. http://dx.doi.org/10.1098/rspa.2004.1404

[37] Kraenkel, R.A., Manikandan, K. and Senthivelan, M. (2013) On Certain New Exact Solutions of a Diffusive PredatorPrey System. Communications in Nonlinear Science and Numerical Simulation, 18, 1269-1274. http://dx.doi.org/10.1016/j.cnsns.2012.09.019

[38] Dehghan, M. and Sabouri, M. (2013) A Legendre Spectral Element Method on a Large Spatial Domain to Solve the Predator-Prey System Modeling Interaction Populations. Applied Mathematical Modeling, 37, 1028-1038. http://dx.doi.org/10.1016/j.apm.2012.03.030 